\title{
Measures undertaken by the dental profession have aided post-COVID-19 recovery
}

\author{
Align Technology, Inc. have published the results of a new survey revealing \\ the measures taken and challenges faced by the dental profession in the UK \\ during and following the COVID-19 lockdown.
}

\section{A} ccording to the report, drawn together from a number of independent sources by Align Technology, the dental profession has risen to the challenge of treating patients throughout the COVID-19 pandemic, with increased safety measures and a rise in virtual consultations compared with preCOVID-19 times.

The report also finds that closing practices again during a potential second wave in the autumn could be detrimental to patient health. In a survey of dentists, therapists, hygienists and nurses working for the 600 mydentist group practices, 96\% stated that lockdown has had an adverse impact on the nation's oral health and that greater access to affordable dental care is needed. Eighty-eight percent of dental health experts surveyed said that the UK's dental health could decline because a lack of routine appointments could lead to preventable dental issues getting worse (research conducted by mydentist amongst 600 dental practices).

Those mydentist professionals surveyed suggested that there were missed opportunities to identify oral cancer which may cause significant long-term health issues. Over three quarters (77\%) of those questioned are especially worried that oral cancers will be missed, and not referred onwards. One thousand seven hundred members of the British Association of Private Dentists concur: nearly 97\% of those surveyed are performing fewer oral cancer screens per day in comparison to their situation pre-lockdown. Oral cancer specialist Professor Mike Lewis reported that where he normally receives 100 oral cancer referrals, over the same period of time, he received only

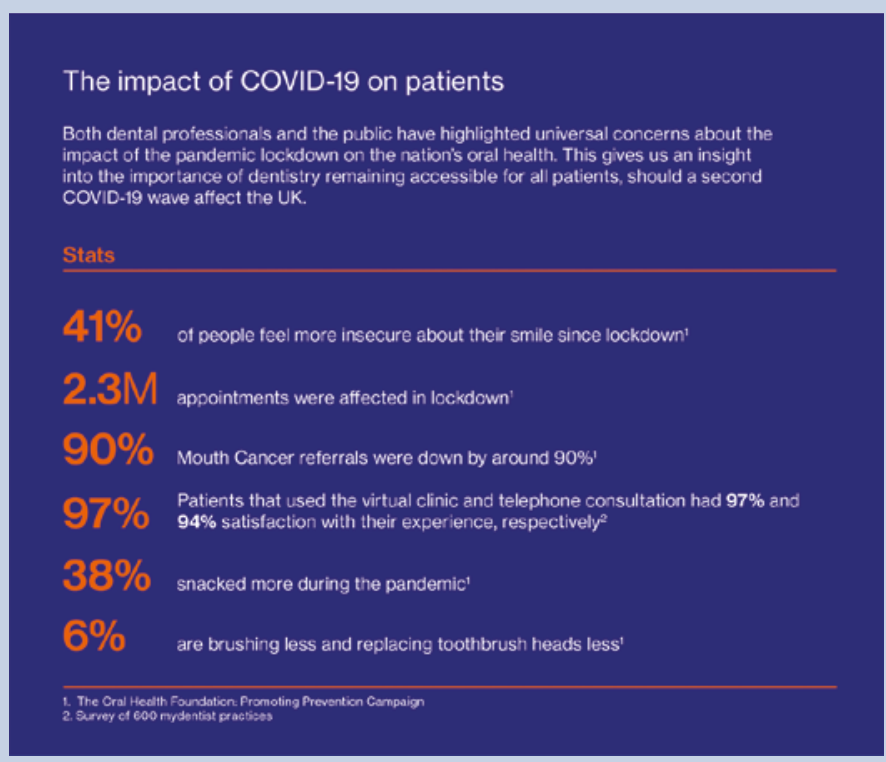

three. Professor Lewis notes this underlines the need to ensure that patients have regular access to dental practices.

Over a quarter $(41 \%)$ of dentists believe it will take between a year and 18 months for the UK's oral health to return to 'normal' after the COVID-19 crisis. The probable rise in the need for implants was a concern that was specifically raised. Dr Guy Laffan, a dentist practising in Wales, advised that he personally extracted ten patients' teeth post lockdown, and felt these could have been saved if his practice had remained open and treatment was accessible. While London based, Dr Gina Vega added that 35-40 of her patients had root canal issues during lockdown. Around half did not meet the criteria to be seen by the dental hubs and some had teeth extracted as a result. Dr Vega expects a surge in the number of implants conducted over the coming months.

Many positive findings also emerged (from mydentist's research amongst 600 dental practices). The report suggests those patients who have been able to connect with a dentist virtually during the lockdown period had a positive experience. Patients who used a virtual clinic or telephone consultation had $97 \%$ and $94 \%$ satisfaction with their experience, respectively. This suggests a future for the use of telehealth in dentistry as an aid for recovery from the coronavirus pandemic.

All dentists questioned agreed or strongly agreed that a teledentistry system would be very useful in saving time. Dr Ben Atkins, president of the Oral Health Foundation, suggests teledentistry has improved the patient experience, with the use of virtual clinics and telephone 


\section{Patient care in a post-COVID world}

As a result of the national lockdown, dental professionals expressed their concerns and highlighted challenges surrounding patient care. If dentistry faces closure during a second lockdown, it is apparent that the nation's oral health could be negatively impacted well beyond the 'new norma'.

Stats

\section{$96 \%$}

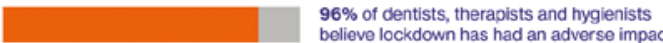
believe lockdown has had an adverse impact on the nation's oral health and that greater to affordable dental care is needod!

\section{$88 \%$}

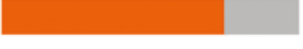

$88 \%$ believed the UK's dental health could decline because of a lack of routine appointments leading to preventative dental issues worsening:

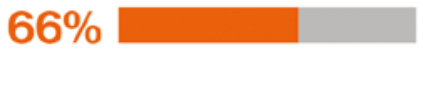

lieve that some patients could put of oing to the dentist to treat minor symptoms, such as toothache and bleeding gums, leading to arger problems in the longer-term?

$41 \%$

$41 \%$ believe it will take between a year and 18-months for the UK's oral health to return to normal' after the Covid-19 crisis.

1. Survey of 600 mydentist prectices

consultations. Dr Atkins recommends that dentists consider increasing their teledentistry offering to help reduce the risk of viral transmission and compliance with social distancing measures.

Adult orthodontics has also been identified as an opportunity for growth for practices post lockdown. Over three quarters (80\%) of orthodontists in a new survey saw an increase in adult patients, and over half (60\%) say this is due to heightened awareness of adult orthodontics. More than $15 \%$ of orthodontists say their adult patients are influenced by celebrities and bloggers. The majority of adult patients $(85 \%)$ are in the $26-55$ age bracket and female (80\%).
According to Dr Mark Cronshaw, President of the Pandora Group [an independent think tank], the dental profession has led the way for stringent safety measures. While protective masks, gloves and clothing have always been the gold standard for patient and doctor protection, during lockdown, this lobbying organisation created a recommended 8-step protocol for reopening safely. This included evidence-based advice about the length of fallow time, air sterilisation, aerosol generating procedures (AGPs), rubber dam, PPE (levels of) suction technologies, and patient pre-treatment checks.

The protocol aims to reduce the risks associated with AGPs down to around $0.1 \%$ risk, and cumulatively reduce the overall risk and impact on the provision of general dental practice. Additionally, while dentists have been advised that they should have a month's worth of PPE in reserve in case of a second wave, no dentists questioned reported concerns about access to PPE, underlining how the profession's forward planning measures are already in place.

Align Technology has committed $\$ 1$ million to the Align Foundation to source and supply personal protective equipment (PPE) and medical supply donations as well as making cash donations to the World Health Organisation in Europe. It is also using its 3D printing technology and manufacturing expertise to produce face shields and medical swaps to support the fight against COVID-19. In addition, the company is supporting campaigns to help dentists and dentistry through sharing best practice, cooperating with leading associations, providing practical support for organisations and developing technologies to support the profession, including introducing virtual solutions to connect doctors and patients.

Visit www.aligntech.com for more information.

\section{Ten years of clinical success}

Trycare Ltd is the UK distributor for Adin implants. With millions sold worldwide and over 40,000 in the UK, Adin implants deliver over ten years of clinical success and are the first choice for many implantologists worldwide.

Adin's spiral design implants are suitable for all indications and exceptional for immediate loading. Their unique thread and tip slices through bone compressing and condensing on the way, offering outstanding primary stability and optimal restorative orientation. If necessary, they can be redirected during placement with little or no reduction of stability, the dual cutting blade edge helping to minimise the osteotomy site and trauma to bone and surrounding soft tissue. This makes them ideal for thinner bone ridges.

Macroscopic grooves on the surface of Adin implant threads double the surface area of most other implants and give better bone-to-implant contact. Whilst their surface treatment has been shown to increase osseointegration. New York University research proved that Adin implants integrate as well, if not better, than Nobel, Straumann and Astra.

For further information visit the Trycare website, www. trycare.co.uk/adin, where you can download the Trycare Surgical Essentials and Adin Catalogues. Alternatively contact your local representative or call 01274885544 .

\section{Centuries of expertise}

Meisinger is a brand that has been pushing boundaries in dentistry since it was established in 1888 . Among its top solutions on the market today is the Master-Pin-Control system, designed specifically to enable the fixation of resorbable, nonresorbable and titanium membranes on local bone.

Developed by Dr Istvan Urban, it provides a reliable and easy-to-use system that offers high-precision fixation, even in compact cortical bone. It also makes removal of pins simple and the whole kit is autoclavable for convenient infection control protocols.

The Master-PinControl system is currently available through J\&S Davis in the UK. Discover how you could improve your implant dentistry by contacting the team today.

For more information on the industry-leading products available from J\&S Davis, visit www.js-davis.co.uk, call 01438 747344 or email jsdsales@js-davis.co.uk. 